\title{
OBSERVACIONES SOBRE UN COMPORTAMIENTO ATIPICO DE RUPPIA MARITIMA L. EN UNA LAGUNA COSTERA TROPICAL *
}

\author{
Martha González Gutiérrez **
}

INTRODUCCIóN

Ruppia maritima L. es una especie de planta acuática caracterizada por una gran tolerancia a la salinidad y una amplia distribución geográfica. Se encuentra principalmente en lagunas costeras, estuarios y bahías; también se puede observar con frecuencia en aguas continentales salobres o dulces.

Ruppia maritima está considerada como una especie cosmopolita (Lawrence, 1951; Arber, 1963; Good, 1964), aunque se encuentra mejor representada en las zonas tropicales y subtropicales (Correl \& Correl, 1972). En Estados Unidos la especie se distribuye en todo el país, encontrándose con mayor frecuencia en la costa atlántica (Muenscher, 1964). En la República Mexicana la especie ha sido colectada en Baja California Norte, Baja California Sur, Sinaloa, Nayarit, Jalisco, Oaxaca, Chiapas, Yucatán, Veracruz, Tamaulipas, San Luis Potosí, Puebla y México. La información sobre la distribución de Ruppia maritima en este país es incompleta, ya que su flora acuática no se ha estudiado a fondo.

En estudios anteriores se ha demostrado que Ruppia maritima presenta variaciones en morfología, fisiología y comportamiento. Estas pueden deberse a la diversidad climática, altitudinal y latitudinal, la naturaleza química del agua y la permanencia o estacionalidad de los cuerpos de agua en los que habita. Dentro de estas variaciones es importante señalar las siguientes:

1) El grado de tolerancia de Ruppia maritima a la salinidad. Este aún no se ha delimitado porque los resultados varían, según el origen del material experimental (Bourn, 1935).

* Este manuscrito es una versión modificada de una tesis para optar al título de biólogo en la Facultad de Ciencias, UNAM.

** Departamento de Botánica, Instituto de Biología. UNAM. Ciudad Universitaria, México 20, D.F. 
2) La especie puede presentarse en ocasiones como una planta anual y a veces como perenne. Conover (1958) la consideró como una hidrófita perenne con época de latencia durante los meses fríos en Massachusetts. Por su parte, Phillips (1960) encontró que presentaba un comportamiento similar en Florida, pero Ungar (1974), la señaló como una especie anual que habitaba charcas estacionales en Texas. En la Bahía de San Francisco, California, Setchell (1924) describió poblaciones anuales y perennes de Ruppia maritima que mostraban además cierta variación fenotípica, de acuerdo con su permanencia en las estaciones estudiadas. Setchell (1924) explicó que la diferencia en comportamiento se debía a que existían dos variedades de Ruppia maritima en una misma localidad.

3) La floración de Ruppia maritima ocurre durante el verano en las zonas templadas. En el laboratorio, sin embargo, puede producir flores durante todo el año (Setchell, 1924; Phillips, 1960).

4. El mecanismo de polinización aún no está bien estudiado. Algunos autores sostienen que ésta se efectúa en la superficie del agua (Roze, 1894; Graves, 1908), mientras que otros opinan que se realiza dentro del agua (Scultorphe, 1967; Gamerro, 1968).

El comportamiento y las variaciones de Ruppia maritima descritas anteriormente se refieren principalmente a observaciones realizadas en zonas templadas. En este estudio se pretende proporcionar información que pueda complementar el conocimiento de la biología de esta especie en zonas tropicales. En forma específica se desea describir su comportamiento en la Laguna de Sontecomapan, Veracruz, y discutir algunos factores que podrían tener relación con su distribución en dicha laguna.

\section{Algunas características del área de estudio}

La Laguna de Sontecomapan está entre $18^{\circ} 30^{\prime}$ lat. N y $94^{\circ} 02^{\prime}$ long. W, en la región de Los Tuxtlas, Veracruz. El clima general del área es Am(f)i, o sea, cálido-húmedo con época seca corta (enero a mayo), con lluvias de monzón y oscilación diurna de temperatura inferior a $5^{\circ} \mathrm{C}$ (García, 1973). Durante los meses de septiembre a mayo, toda la costa del Golfo de México es afectada por vientos huracanados que vienen del norte (Fig. 1).

Esta laguna que mide casi $9 \mathrm{~km}$ de longitud se puede dividir en dos regiones de acuerdo con Ringuelet (1962). La primera es semejante a una albufera; la otra se puede considerar como un estuario (Fig. 2). En ambos casos existen ambientes mixohalinos (mezcla de aguas dulces y marinas) aunque en 


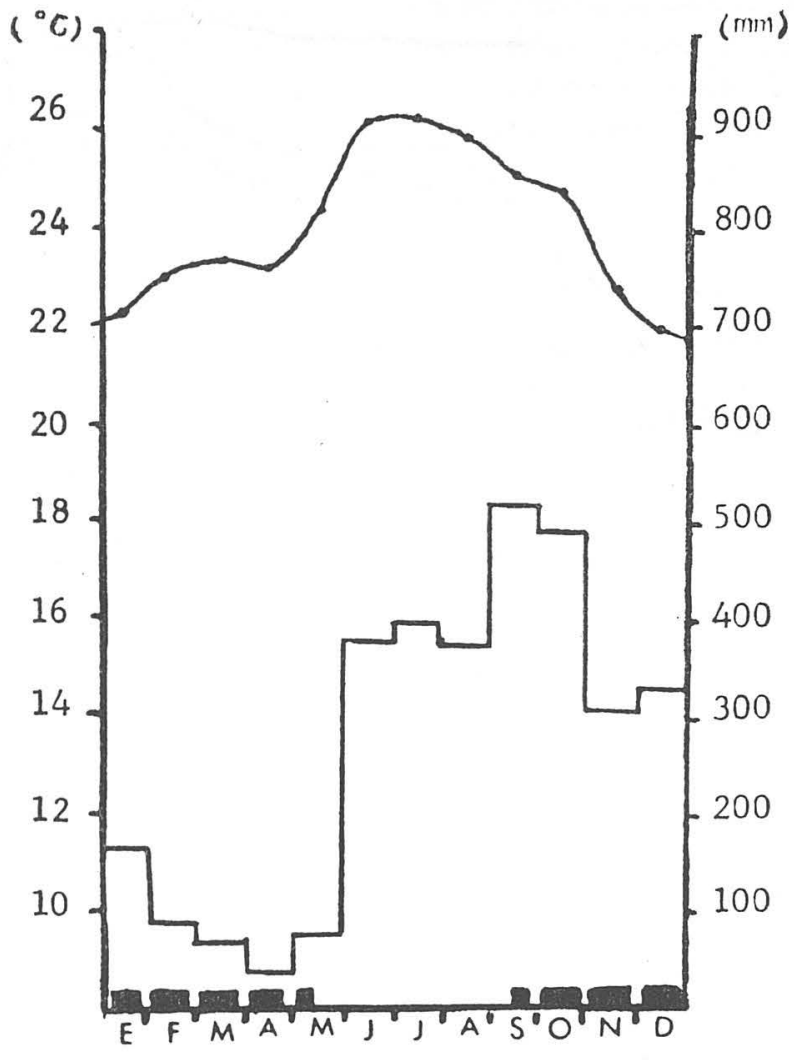

Fig. 1. Climograma de la Estación Meteorológica del Faro de Zapotitlán, Ver. En la parte inferior, las barras oscuras marcan la época de "nortes". 


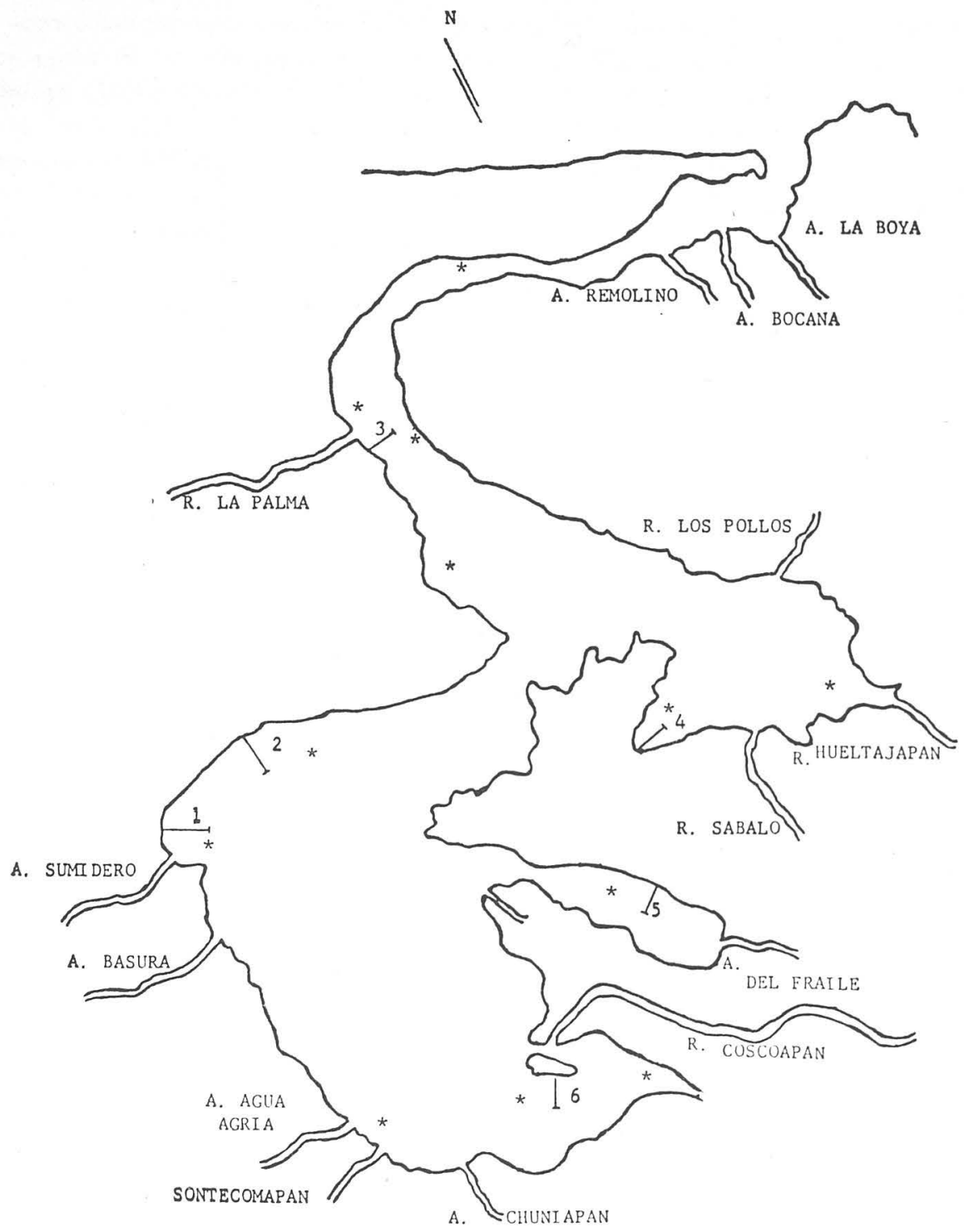

Fig. 2. Mapa general de la Laguna de Sontecomapan. Las estaciones de muestreo están marcadas con números. Los asteriscos señalan las zonas donde se colectó el agua para hacer evaluaciones de salinidad. 
la albufera las aguas son "lénticas" (quietas) y en el estuario "lóticas" (móviles).

La profundidad promedio en la albufera es de 1 a $1.5 \mathrm{~m}$, dependiendo del nivel de mareas, pero se puede llegar a más de $2 \mathrm{~m}$ en los canales que la atraviesan. El estuario presenta una pendiente de $2 \mathrm{~m}$ en la zona de contacto con la albufera aumentan de 5 a $6 \mathrm{~m}$ en la desembocadura al mar. El agua en la albufera presenta una visibilidad muy baja por tener mucho material en suspensión, mientras que en el estuario, la transparencia del agua aumenta gradualmente hacia la bocana.

\section{Metodología}

En abril de 1974, se hizo una visita preliminar a la Laguna de Sontecomapan y se elaboró un mapa de distribución de "manchones" de Ruppia maritima, su extensión y grado de desarrollo. En base a esa información se escogieron seis estaciones permanentes de muestreo, las cuales presentaban diferencias en orientación, relación a afluentes cercanos y tipo de sustrato (Fig. 2). De acuerdo con estos tres factores, las estaciones quedaron caracterizadas como se indica en la Tabla 1.

\section{TABLA 1}

Caracteristicas de las estaciones de muestreo

\begin{tabular}{clll}
\hline Estación & Localidad $^{1}$ & Afluentes cercanos $^{2}$ & Sustrato $^{3}$ \\
\hline 1 & expuesta & si & migajón-arenoso \\
2 & expuesta & no & migajón-arenoso \\
3 & protegida & si & arenoso \\
4 & expuesta & si & migajón-arenoso \\
5 & protegida & no & migajón-arenoso \\
6 & expuesta & no & arena-migajosa \\
\hline
\end{tabular}

1 Se consideró "protegida" a la localidad ubicada en pequeñas bahías o meandros y "expuesta" donde el litoral sigue en línea recta.

2 Afirmativo, cuando el afluente estaba dentro de un radio de quinientos metros.

3 Análisis, según Sampat (1973). 
En visitas mensuales a la laguna se tomaron lecturas sobre la posición y abundancia relativa de los "manchones". En cada estación permanente se hicieron observaciones sobre profundidad, altura de la planta, enterramiento del rizoma, estado general de la planta, presencia de flores o frutos y forma del "manchón". Para hacer evaluaciones con respecto a la productividad de los "manchones" se obtuvieron los valores de biomasa (peso seco por $\mathrm{m}^{2}$ ).

Durante mayo, septiembre y diciembre se trazaron transectos de la playa hacia el centro de la laguna en cada una de las estaciones de muestreo. Sobre cada transecto se tomaron tres muestras de sedimento para efectuar los siguientes análisis:

1) $\mathrm{pH}$ : método electrométrico del electrodo de vidrio (Contin, 1973).

2) Porcentaje de cloruros: método de titulación de Mohr (Contin, 1973).

3) Porcentaje de materia orgánica: método de digestión de dicromato ácido (Contin, 1973).

4) Textura: método de clasificación de partículas, según los sistemas del Departamento de Agricultura de los Estados Unidos y la Sociedad Internacional de Ciencias del Suelo (Sampat, 1973).

Para estudios de salinidad del agua se hicieron muestreos mensuales en doce estaciones seleccionadas (Fig. 2). El valor de la salinidad se obtuvo empleando un refractómetro American Optical Corporation N No An 169.

\section{Resultados}

Aunque siempre se encontraron "manchones" de Ruppia maritima en la laguna, éstos ocupaban localidades variables con una marcada asincronía en sus fases de desarrollo. En la Figura 3, se esquematiza la posición y extensión relativa de los "manchones" de la especie durante cuatro meses del año y en la Figura 4, se presentan los valores de biomasa obtenidos para las seis estaciones permanentes de muestreo. Durante mayo y agosto los "manchones" mejor desarrollados se encontraban ocupando la región de la albufera. En mayo ocupaban principalmente la costa Oeste (estaciones 1 y 2) y en agosto, la Este (estaciones 4, 5 y 6). En diciembre, los "manchones" mejor desarrollados se encontraban en la región del estuario (estación 3) ocupando dos tercios de su costa Oeste. Sin embargo, en abril sólo se pudieron observar pequeños "manchones" que empezaban a expanderse (Fig. 4, estaciones 4, 5 y 6 ).

Los análisis efectuados sobre muestras de agua y suelo revelaron lo siguiente:

La salinidad del agua y sedimento (Tabla 2) mostraron valores bajos durante la época de lluvias y altos en época de secas (cf. Fig. 1). Sin embargo, 


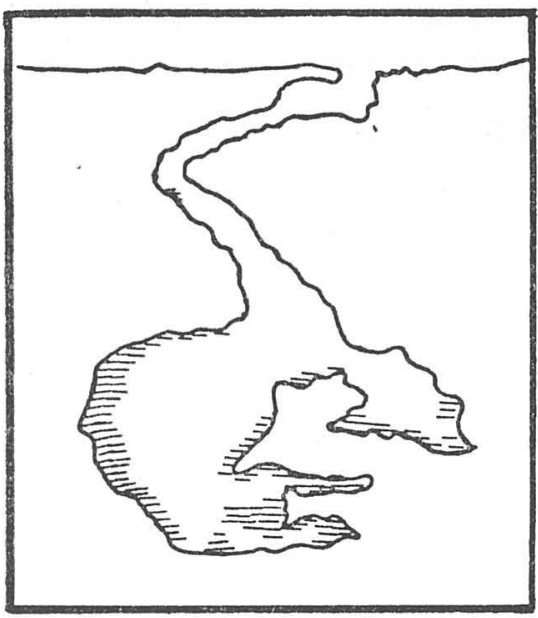

MAYO

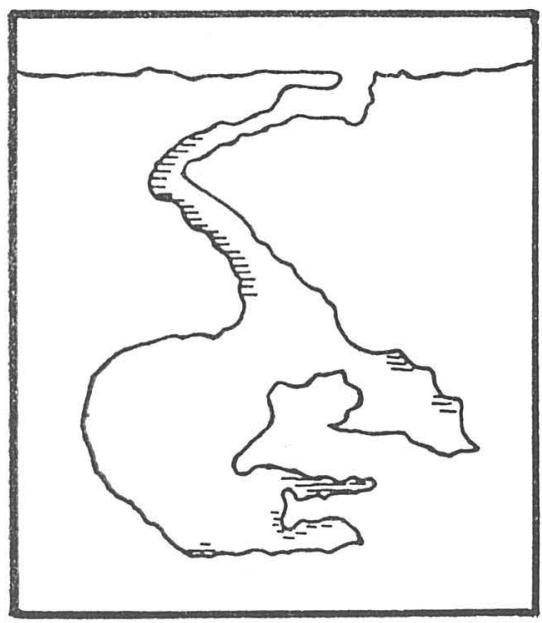

DICIEMBRE

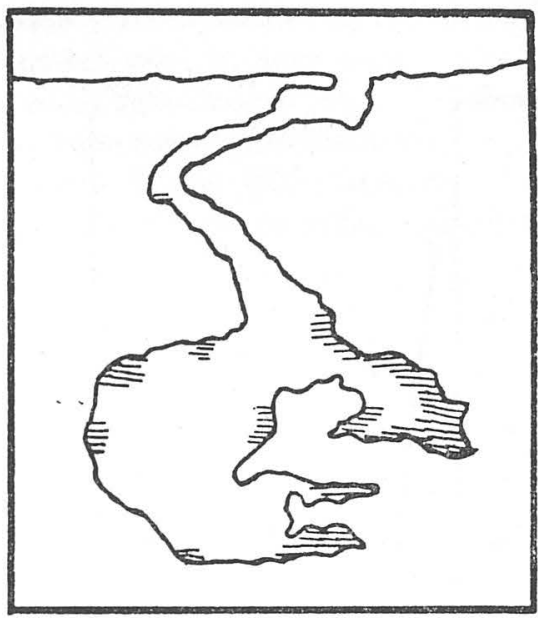

AGOSTO

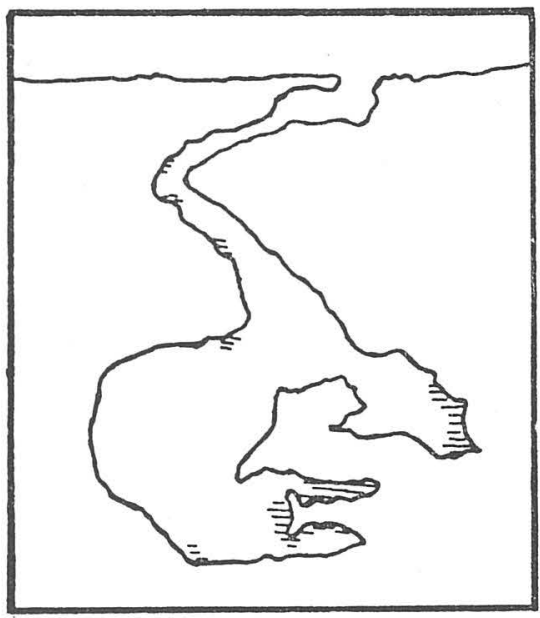

ABRIL

Fig. 3. Distribución de los "manchones" de Ruppia maritima (zonas sombreadas) en la Laguna de Sontecomapan durante el año de estudio. 

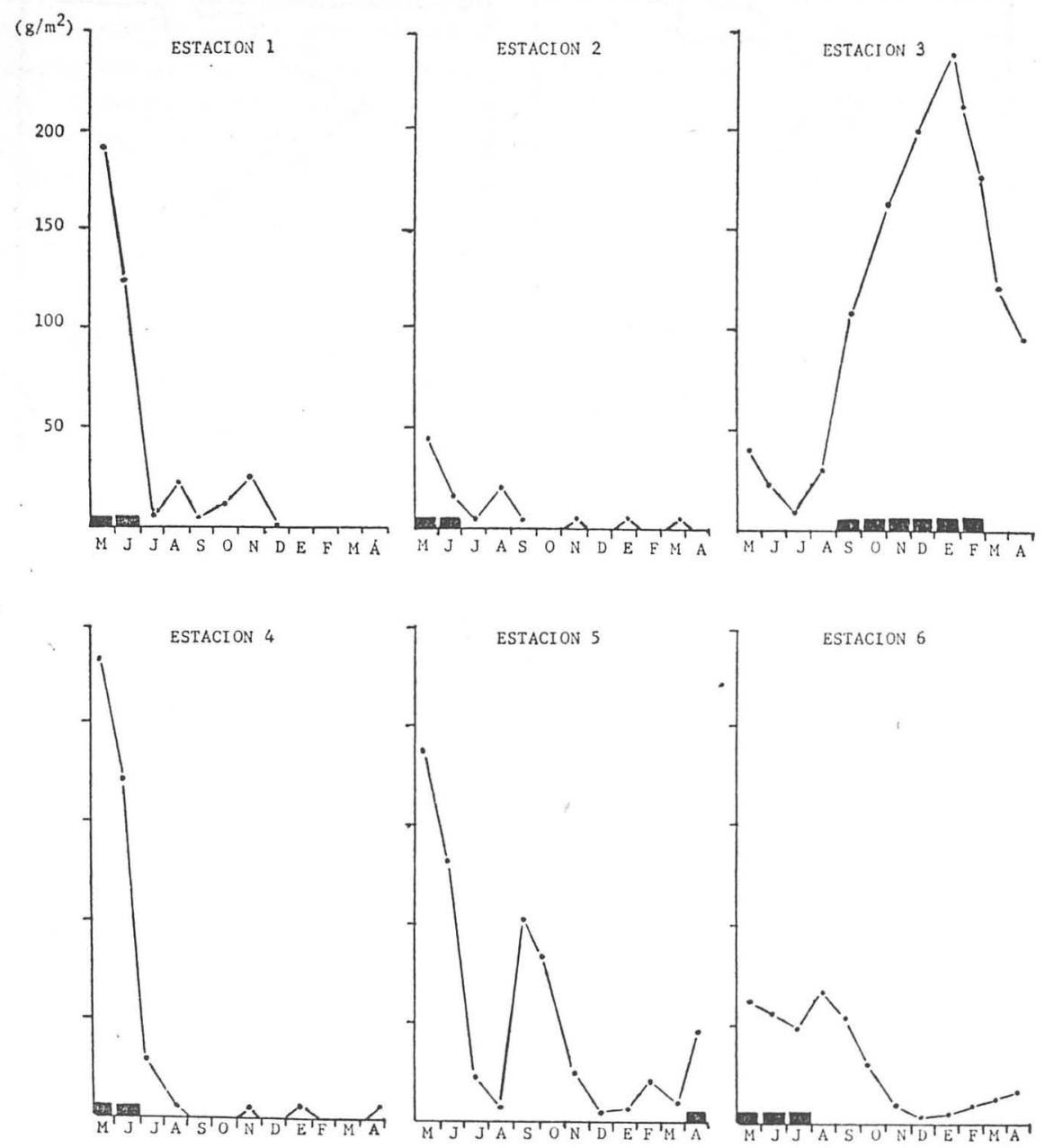

Fig. 4. Valores de biomasa de los "manchones" en las seis estaciones de muestreo. Las barras oscuras señalan la presencia de floración y fructificación. 
esto no pareció afectar a Ruppia. La floración y fructificación ocurrieron en la laguna, tanto en agua dulce (mes de diciembre) como en agua salada (mes de mayo) (cf. Tabla 2 y Fig. 4). Aunque no se ha delimitado el grado de tolerancia de esta especie a la salinidad, existe la opinión entre los distintos autores que se trata de una especie eurihalina (Graves, 1908; Bourn, 1935; Conover, 1958; Arber, 1963; Fasset, 1969), ya sea en aguas con cloruro de sodio, carbonatos, fosfatos o compuestos de magnesio.

TABLA 2

Porcentaje de salinidad en agua y suelo

\begin{tabular}{lcclcc}
\hline \multicolumn{1}{c}{ Mes } & Agua & Suelo & \multicolumn{1}{c}{ Mes } & Agua & Suelo \\
\hline Mayo & 1.43 & 2.6 & Noviembre & 0.43 & $\ldots$ \\
Junio & 0.83 & $\ldots$ & Diciembre & 0.35 & 0.88 \\
Julio & 0.14 & $\ldots$ & Enero & 0.30 & $\ldots$ \\
Agosto & 0.31 & $\ldots$ & Febrero & 0.58 & $\ldots$ \\
Septiembre & 0.55 & 0.94 & Marzo & 1.18 & $\ldots$ \\
Octubre & 0.57 & $\ldots$ & Abril & 1.18 & $\ldots$ \\
\hline
\end{tabular}

Se encontraron tres distintos tipos de suelo de acuerdo con su textura (Tabla 3). Ruppia mostró diferencias en su comportamiento, dependiendo del tipo de sustrato en el que se arraigaba. En los suelos de tipo arenoso o areno-migajoso los rizomas se enterraban a $3 \mathrm{~cm}$ de profundidad y los "manchones" eran perennes, mientras que en los de tipo migajón-arenoso, los rizomas se enterraban a 1.5 o $2 \mathrm{~cm}$ y los "manchones" desaparecieron en alguna época del año. En las zonas donde existen sustratos de tipo arenoso y areno-migajoso el suelo es firme, al grado que se puede caminar sobre él sin hundirse; en las zonas con sustratos del tipo migajón-arenoso el sustrato es flojo y no se puede caminar sin hundirse hasta las rodillas en el lodo.

Cuando los "manchones" de Ruppia desaparecían de alguna localidad, e] porcentaje de materia orgánica del suelo aumentaba, mientras que los valores de $\mathrm{pH}$ bajaban (Tabla 4 estaciones 1, 2 y 4). En donde los "manchones" no desaparecían el pH era neutro o ligeramente ácido (no menor de 6.0). En Kansas, Ungar (1974) encontró que las poblaciones de Ruppia ocupaban zonas donde el pH oscilaba entre 6.8 y 8.7 con un promedio de 7.7, y Setchell (1924) observó que cuando la planta está en su mayor actividad, durante el día tiene un efecto 
BOLETIN DE LA SOCIEDAD BOTANICA DE MEXICO N 37,1977

TABLA 3

Textura del sustrato en las estaciones permanentes de muestreo

\begin{tabular}{|c|c|c|c|c|c|}
\hline Estación & Porcentaje & Mayo & Septiembre & Diciembre & Textura \\
\hline 1 & $\begin{array}{l}\text { arena } \\
\text { arcilla } \\
\text { limo }\end{array}$ & $\begin{array}{r}62.38 \\
1.42 \\
38.52\end{array}$ & $\begin{array}{r}70.96 \\
0.71 \\
28.33\end{array}$ & $\begin{array}{r}65.72 \\
4.28 \\
30.00\end{array}$ & $\begin{array}{r}\text { Migajón- } \\
\text { arenoso }\end{array}$ \\
\hline 2 & $\begin{array}{l}\text { arena } \\
\text { arcilla } \\
\text { limo }\end{array}$ & $\begin{array}{r}66.95 \\
2.13 \\
30.71\end{array}$ & $\begin{array}{r}73.20 \\
1.42 \\
25.38\end{array}$ & $\begin{array}{r}77.15 \\
0.00 \\
22.85\end{array}$ & $\begin{array}{r}\text { Migajón- } \\
\text { arenoso }\end{array}$ \\
\hline 3 & $\begin{array}{l}\text { arena } \\
\text { arcilla } \\
\text { limo }\end{array}$ & $\begin{array}{r}87.13 \\
1.42 \\
11.42\end{array}$ & $\begin{array}{r}91.91 \\
0.00 \\
8.09\end{array}$ & $\begin{array}{r}87.96 \\
2.61 \\
9.28\end{array}$ & Arenoso \\
\hline 4 & $\begin{array}{l}\text { arena } \\
\text { arcilla } \\
\text { limo }\end{array}$ & $\begin{array}{r}65.71 \\
3.32 \\
30.95\end{array}$ & $\begin{array}{r}57.15 \\
0.70 \\
42.42\end{array}$ & $\begin{array}{r}64.29 \\
4.28 \\
31.43\end{array}$ & $\begin{array}{l}\text { Migajón- } \\
\text { arenoso }\end{array}$ \\
\hline 5 & $\begin{array}{l}\text { arena } \\
\text { arcilla } \\
\text { limo }\end{array}$ & $\begin{array}{r}63.81 \\
0.47 \\
35.71\end{array}$ & $\begin{array}{r}65.71 \\
0.47 \\
30.95\end{array}$ & $\begin{array}{r}65.42 \\
6.66 \\
28.09\end{array}$ & $\begin{array}{r}\text { Migajón- } \\
\text { arenoso }\end{array}$ \\
\hline 6 & $\begin{array}{l}\text { arena } \\
\text { arcilla } \\
\text { limo }\end{array}$ & $\begin{array}{r}76.91 \\
0.93 \\
22.14\end{array}$ & $\begin{array}{r}84.29 \\
0.00 \\
15.71\end{array}$ & $\begin{array}{r}83.33 \\
3.56 \\
9.76\end{array}$ & $\begin{array}{r}\text { Arena- } \\
\text { migajosa }\end{array}$ \\
\hline
\end{tabular}

basificador sobre el medio. Este autor encontró a la especie en sustratos cuyo $\mathrm{pH}$ variaba de 7.4 a 9.6. Es posible que un $\mathrm{pH}$ muy ácido no permita el establecimiento de rizomas de Ruppia en el suelo. La materia orgánica proviene principalmente del manglar que rodea a la laguna en casi todo su borde y también de los "manchones" de Ruppia. Esto último ocurre especialmente al inicio de la épaca de lluvias, ya que los afluentes que llegan a la laguna traen consigo grandes cantidades de sedimento que entierran a los "manchones", provocando la muerte de las plantas. Este material en descomposición baja el $\mathrm{pH}$ y es posible que este fenómeno contribuya a evitar el establecimiento de nuevos "manchones" de Ruppia en algunas zonas de la laguna en determinadas épocas. 
TABLA 4

Valores promedio de pH y materia orgánica en el sedimento

\begin{tabular}{|c|c|c|c|c|c|c|}
\hline \multirow{2}{*}{ Estación } & \multicolumn{2}{|c|}{ Mayo } & \multicolumn{2}{|c|}{ Septiembre } & \multicolumn{2}{|c|}{ Diciembre } \\
\hline & $\mathrm{pH}$ & M.O. & $\mathrm{pH}$ & M.O. & $\mathrm{pH}$ & M.o. \\
\hline 1 & 6.9 & 30.0 & 5.3 & 35.3 & 5.7 & 40.0 \\
\hline 2 & 6.7 & 8.7 & 5.9 & 19.0 & 6.0 & 30.0 \\
\hline 3 & 7.0 & 8.6 & 6.5 & 14.0 & 7.0 & 11.3 \\
\hline 4 & 6.4 & 11.6 & 5.1 & 43.0 & 4.9 & 40.0 \\
\hline 5 & 6.5 & 9.8 & 6.2 & 24.7 & 7.0 & 19.0 \\
\hline 6 & 6.8 & 7.5 & 6.0 & 13.7 & 7.0 & 14.3 \\
\hline
\end{tabular}

Como resultado de los transectos realizados, las visitas mensuales y las observaciones de campo, se piensa que el desarrollo de los "manchones" de Ruppia maritima en la Laguna de Sontecomapan puede consistir de cuatro fases (Fig. 5) : 1) dispersión por propágulos vegetativos, 2) establecimiento de "manchones", 3) crecimiento explosivo y 4) reducción de "manchones". Estas se discuten a continuación:

1) Dispersión por propágulos vegetativos. Los tallos y rizomas de Ruppia maritima, además de ser muy frágiles, suelen presentar puntos de abscisión. Frecuentemente se puede encontrar partes de la planta, originalmente arraigadas al sustrato, flotando a la deriva. Este material flotante, constituido principalmente por tallos, llega a producir raíces después de algún tiempo. Cuando éstos se depositan en zonas someras y tranquilas, enraizan y empiezan a crecer sobre el sustrato. En algunos casos, los rizomas recién enraizados no logran pasar a la siguiente etapa, debido a la acción de las corrientes producidas por los "nortes" y a los constantes aportes de sedimento bajo los cuales sucumben los rizomas (estaciones 2 y 4, Fig. 4 a partir de septiembre).

2) Establecimiento de "manchones". Los rizomas recién enraizados forman pequeños "manchones" más o menos circulares que se extienden rápidamente y avanzan de zonas de menor profundidad (donde el agua es clara y estancada) a zonas más profundas (con aguas turbias). Esta fase se manifiesta por la formación de rizomas únicamente y se puede observar en la gráfica de biomasa de la estación 6 (Fig. 4) durante los meses de diciembre a abril. En esta etapa los valores aumentan de una forma moderada.

3) Crecimiento explosivo. Esta fase se manifiesta por la formación de tallos 


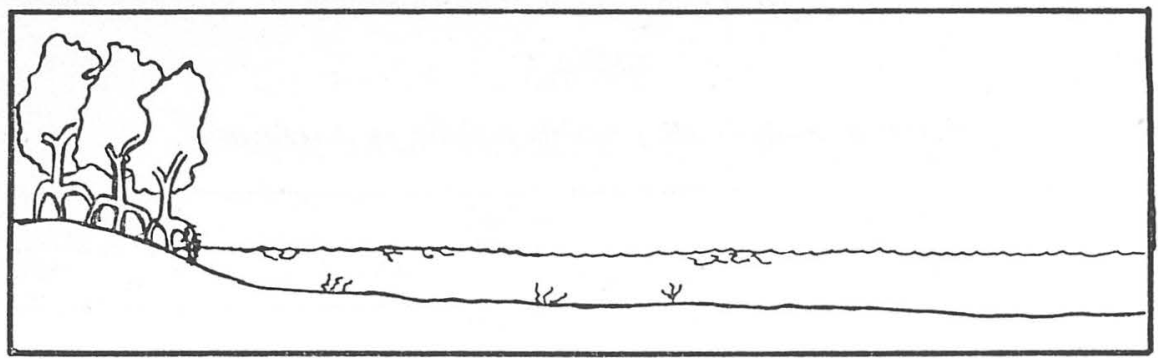

PRIMERA FASE "DISPERSION POR PROPAGULOS VEGETATIVOS"

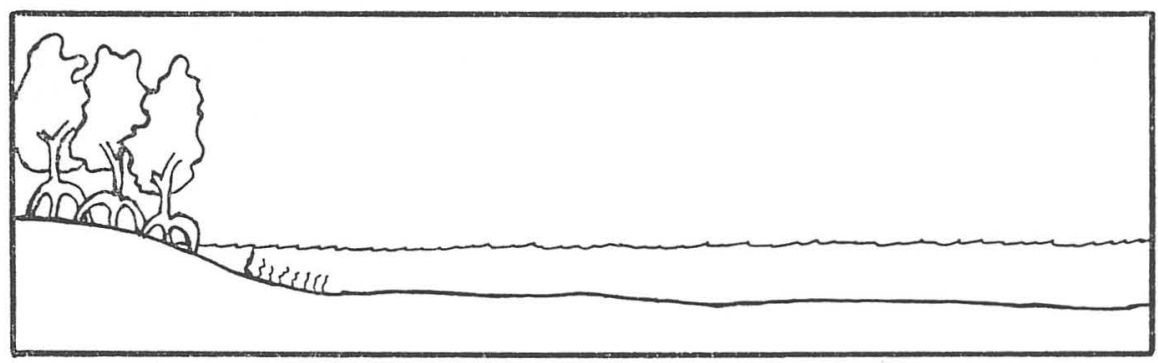

SEGUNDA FASE "ESTABLECIMIENTO DE MANCHONES"

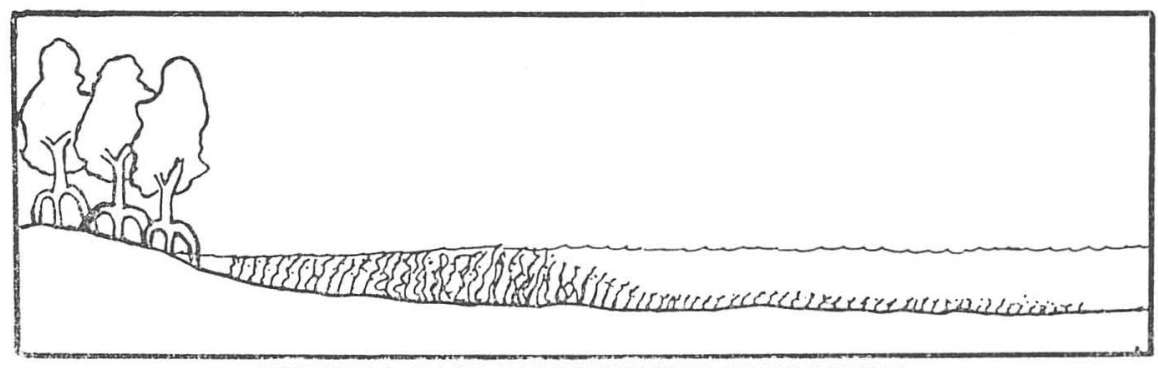

TERCERA FASE "CRECIMIENTO EXPLOSIVO"

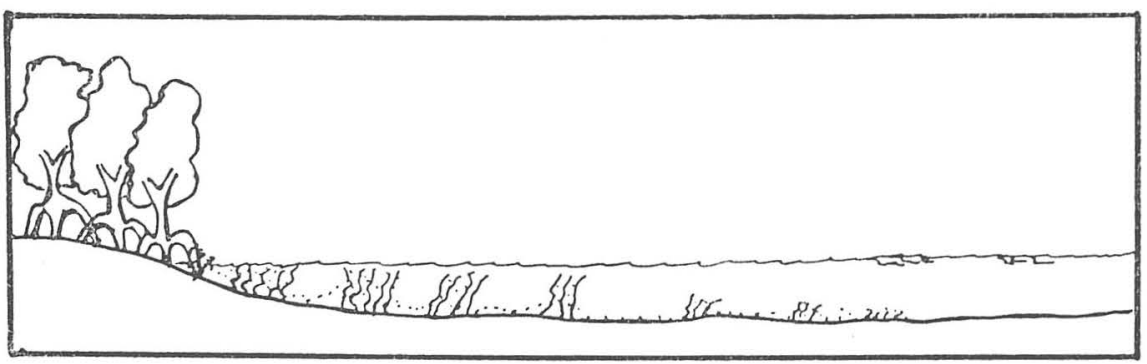

CUARTA FASE "REDUCCION DE MANCHONES"

Fig. 5. Perfil diagramático de las fases de desarrollo de los "manchones" de Ruppia maritima en la Laguna de Sontecomapan. 
a partir de los rizomas, los cuales llegan a la superficie del agua. Los "manchones" en esta etapa son muy densos, suelen presentar flores y frutos y ocupan extensas áreas en el litoral y hacia el centro de la laguna. A cierta profundidad (más de $1.30 \mathrm{~m}$ ) la planta sólo crece por la extensión de rizomas en el fondo y no por la producción de tallos. Phillips (1960), por su parte, ha reportado poblaciones de Ruppia ocupando zonas entre 0.70 a $1.40 \mathrm{~m}$ de profundidad, dependiendo del nivel de mareas y considera que a profundidades mayores, la turbidez del agua limita su existencia.

4) Reducción de "manchones". Este fenómeno se realiza por el desprendimiento masivo de tallos que, en la mayoría de los casos, lleva a la total destrucción del "manchón". Los tallos quedan flotando a la deriva y algunos de ellos pueden reiniciar el ciclo en alguna otra parte de la laguna.

Las fases de crecimiento explosivo y reducción son las que más claramente se reconocen en la Figura 4 y corresponden a los fuertes aumentos y descensos, respectivamente.

La floración se inicia en los "manchones" de Ruppia que han entrado en la fase de crecimiento explosivo y termina con la reducción de "manchones". Una vez iniciada la floración, es necesario esperar una semana para poder encontrar frutos. Aunque no se hicieron cuantificaciones con respecto a la producción de flores y frutos en los "manchones" estudiados, se puede decir que la producción de ambos resultó muy abundante durante el año de estudio.

No obstante que se puso cuidado especial al buscar plántulas de Ruppia en la laguna, nunca fue posible encontrarlas por las precarias condiciones de visibilidad y por su pequeño tamaño $(2 \mathrm{~cm})$. Por otro lado, cuando el rizoma crece, se fragmenta y en esta etapa no es posible distinguir a un individuo producido por semilla, de otro producido de modo vegetativo. Arber (1963) y Scultorphe (1967) coinciden en señalar que aunque los mecanismos de floración y fructificación suelen ser muy abundantes en la mayoría de las plantas acuáticas, éstas se reproducen principalmente de modo vegetativo. En la Laguna de Sontecomapan Ruppia maritima presentó una reproducción vegetativa muy intensa (cf. Fig. 5).

\section{Discusión}

La principal diferencia que existe entre los resultados obtenidos durante este estudio y los que se tienen en la literatura, es que mientras en otros lugares la especie presenta ciclos de vida estacionales, en la Laguna de Sontecomapan los "manchones" mostraron una asincronía en sus fases de desarrollo con la época del año (Figs. 3 y 4). Esto se debe a que la mayoría de los estudios sobre la 
especie se han desarrollado en ambientes templados en los cuales algunos factores climáticos, tales como la temperatura y el fotoperíodo, son muy marcados. En la Laguna de Sontecomapan se tiene un clima tropical donde las temperaturas y los fotoperíodos son más moderados.

De acuerdo con Setchell (1924) y Phillips (1960), Ruppia maritima es muy sensible a los cambios de temperatura. La germinación ocurre entre los $10^{\circ} \mathrm{y}$ $18^{\circ} \mathrm{C}$; entre los $18^{\circ}$ y $25^{\circ} \mathrm{C}$ se efectúa un crecimiento vegetativo intenso junto con floración y fructificación, pero por arriba de $25^{\circ}$ y por debajo de $18^{\circ} \mathrm{C}$ el crecimiento se reduce y en ocasiones se detiene. Los autores antes mencionados señalan que la floración y la latencia están reguladas también por ciertos fotoperíodos (desgraciadamente no mencionan cuáles son). En la Laguna de Sontecomapan la floración se realiza durante casi todo el año, aunque en diferentes "manchones" y no se presenta latencia (cf. Fig. 4). La temperatura parece ser, por lo tanto, uno de los factores más importantes en la regulación de las distintas fases de desarrollo de la planta.

Además de la temperatura, Ruppia responde a otros factores ambientales. Dentro de éstos, los más importantes parecen ser la turbidez, las corrientes y la salinidad.

La turbidez y las corrientes actúan como limitantes en el establecimiento de la especie en la laguna. La turbidez evita que se implante a ciertas profundidades (Phillips, 1960) debido a que es menor la cantidad y calidad de luz que puede llegar al fondo. Debido a que Ruppia maritima es una hidrófita sumergida que tiene un sistema de anclaje poco eficiente (Arber, 1963), suele ser arrasada por corrientes fuertes, especialmente en sustratos "flojos" (migajón-arenosos).

La salinidad es otro factor importante en el desarrollo de la especie en el área. Sin embargo, el efecto de ésta no es directo sobre la planta. Hartog (1970) y Hartog \& Segal (1964) consideran que Ruppia maritima tiene poca capacidad de competencia y que las fluctuaciones en salinidad eliminan del medio, especies más estenohalinas con una mayor capacidad competitiva.

En la Laguna de Sontecomapan el crecimiento de los "manchones" de Ruppia maritima disminuye la velocidad de las corrientes y en consecuencia aumenta la transparencia del agua. De esta forma, se invaden grandes áreas en el litoral y hacia el centro de la laguna. La formación de tallos verticales da una mayor estabilidad a las zonas invadidas, donde la floración y fructificación se realizan en forma abundante. En esta fase es probable que el efecto de temperaturas, muy altas o muy bajas, provoque una disminución o suspensión total del crecimiento. Posteriormente, el rompimiento de los tallos hace que se dejen de controlar las corrientes, que aumente la turbidez y haya gran mortalidad entre las plantas. La 
materia orgánica en descomposición, que resulta de estos "manchones", acidifica el pH del suelo, con lo que la reducción y destrucción de los mismos se realizan de forma casi siempre irreversible, principalmente en los sustratos migajón-ạrenosos.

Los resultados obtenidos en este estudio permiten suponer que Ruppia maritima puede llegar a controlar algunos factores, tales como la turbidez y las corrientes, que limitan su existencia en algunas partes de la laguna. El desarrollo logrado parece depender de la temperatura. Si la temperatura fuera óptima durante todo el año, la planta podría permanecer en su máxima actividad el mismo tiempo (Setchell, 1924, ha logrado esto en condiciones experimentales). Esto no significa, sin embargo, que sea la temperatura el factor responsable del desarrollo asincrónico de los "manchones", sino de la acción conjunta de varios de ellos, donde la temperatura ocupa un lugar preponderante.

\section{Agradecimientos}

Se agradece la ayuda del M. en C. Antonio Lot Helgueras por la dirección de la tesis y a los doctores Claudio Delgadillo M. y José Sarukhán K., por la revisión crítica y las sugerencias dadas para mejorar el manuscrito.

\section{RESUMEN}

Ruppia maritima está presente en la Laguna de Sontecomapan durante todo el año. Su distribución en la laguna parece depender de la acción conjunta de varios factores. Entre ellos, turbidez, corrientes y $\mathrm{pH}$ actúan como limitantes. En contraste, suelos con altos porcentajes de arena, fuertes fluctuaciones en salinidad del agua y temperaturas entre $18^{\circ}$ y $25^{\circ} \mathrm{C}$ parecen favorecer el desarrollo de los "manchones" de Ruppia maritima. La combinación de estos factores en diferentes sitios de la laguna tiene como resultado el desarrollo asincrónico de los "manchones".

\section{Abstract}

Ruppia maritima is found in Laguna de Sontecomapan throughout the year. Its distribution within the lagoon boundaries is apparently regulated by several factors. Turbidity, currents and $\mathrm{pH}$ appear to restrict bed growth. In contrast, high proportions of sand in the soil, sirong fluctuations in water salinity and temperatures ranging between $18^{\circ}-25^{\circ} \mathrm{C}$ are favorable for its development. In- 
teractions of all these factors in different sites in the lagoon produce an asyncrony of development of Ruppia beds during the year.

\section{BIBLIOGRAFIA}

Arber, A. 1963. Water Plants. J. Kramer and Weighein. New York. 436 p.

Bourn, W.S. 1935. Sea water tolerance of Ruppia maritima L. Contr. Boyce Thompson Inst. $7(3): 249-255$.

Conover, J.T. 1958. Seasonal growth of benthic marine plants as related to environmental factors in an estuary. Inst. Sci. Univ. Texas. 5:97-147.

Contrn, A. 1973. Investigación de suelos. Ed. Trillas. México. 98 p.

Correl, S.D. \& H.B. Corret. 1972. Aquatic and wetland plants of Southwestern United States. Environmental Protection Agency. Washington, D.C. 1800 p.

Fasset, N.C. 1969. A manual of aquatic plants. Univ. Wisconsin Press. Madison, Milwakee \& London. 416 p.

Gamerro, J.C. 1968. Observaciones sobre la biología floral y morfología de la Potamogetonaceae Ruppia cirrhosa. Darwiniana 14(4) :575-607.

García, E. 1973. Modificaciones al sistema de clasificación climática de Köppen. Instituto de Geografía, UNAM. 245 p.

Good, R. 1964. The geography of the flowering plants. Spottiswode, Ballantyne \& Co. Ltd. London. $518 \mathrm{p}$.

Graves, A.H. 1908. The morphology of Ruppia maritima L. Trans. Conneticut Acad. Arts. Sci. 14:61-169.

Hartog, C.D. 1970. The sea grasses of the world. North-Holland Publishing Co. Amsterdam. 275 p.

- \& S. Segal. 1964. A new classification of the water plant communities. Acta Bot. Neerl. 13:367-393.

Lawrence, G.A.M. 1951. Taxonomy of vascular plants. McMillan Co. New York. 416 p.

Muenscher, W.C. 1964. Aquatic plants of the United States. Cornell Univ. Press. Ithaca, New York. $374 \mathrm{p}$.

Phillips, R.C. 1960. Observations in the ecology and distribution of the Florida sea-grasses. Professional Paper Ser. Florida State Board of Conservation Marine Lab. 2:58-63.

Ringuelet, R.A. 1962. Ecología acuática continental. EUDEBA. Buenos Aires. 183 p.

Roze, M.E. 1894. Recherchez sur les Ruppia. Bull. Soc. Bot. France. 41:466-480.

Sampat, A.G. 1973. Fisica de suelos, principios y aplicaciones. Ed. Limusa-Wiley, S.A. México. $370 \mathrm{p}$.

Scultorphe, C.D. 1967. The biology of aquatic vascular plants. Edward Arnold Publ. Ltd. London. $632 \mathrm{p}$.

Setchell, W.A. 1924. Ruppia and its environmental factors. Botany, 10:286-289.

Ungar, I.A. 1974. Inland halophytes of the United States. EN : ReIlmold, R.J. \& W.H. QueEN. Ecology of halophytes. Academic Press, Inc., pp. 235-305. 